\title{
Carbon Nanoparticles Based Electrochemical Biosensor Strip for Detection of Japanese Encephalitis Virus
}

\author{
Huat Choi Lai, ${ }^{1}$ Suk Fun Chin, ${ }^{1}$ Suh Cem Pang, \\ Magdline Sia Henry Sum, ${ }^{2}$ and David Perera ${ }^{2}$ \\ ${ }^{1}$ Department of Chemistry, Faculty of Resource Science and Technology, Universiti Malaysia Sarawak, 94300 Kota Samarahan, \\ Sarawak, Malaysia \\ ${ }^{2}$ Institute of Health and Community Medicine, Universiti Malaysia Sarawak, 94300 Kota Samarahan, Sarawak, Malaysia
}

Correspondence should be addressed to Suk Fun Chin; sukfunchin@gmail.com

Received 28 July 2017; Revised 27 October 2017; Accepted 13 November 2017; Published 18 December 2017

Academic Editor: Rajesh R. Naik

Copyright (C) 2017 Huat Choi Lai et al. This is an open access article distributed under the Creative Commons Attribution License, which permits unrestricted use, distribution, and reproduction in any medium, provided the original work is properly cited.

\begin{abstract}
We reported a disposable and sensitive electrochemical biosensor strip based on carbon nanoparticles modified screen-printed carbon electrode (SPCE) for rapid and sensitive detection of Japanese Encephalitis Virus (JEV). Amino group functionalized carbon nanoparticles were prepared from preformed chitosan nanoparticles. Japanese Encephalitis Virus (JEV) antibody was immobilized onto the surfaces of carbon nanoparticles through amide bonds formation between amino groups of carbon nanoparticles and carboxylic groups of JEV antibody. The analytical performance of SPCE electrochemical biosensor strip was characterized using cyclic voltammetry (CV) and electrochemical impedance spectroscopy (EIS). SPCE electrochemical biosensor strip exhibited a linear detection range of $1-20 \mathrm{ngmL}^{-1}$ with a low detection limit of $0.36 \mathrm{ngmL}^{-1}$ (at $\mathrm{S} / \mathrm{N}=3$ ) for JEV, detection sensitivity was $0.024 \mathrm{ngmL}^{-1}$ for JEV, and analysis results were obtainable within 10 minutes. The potential clinical application of this SPCE electrochemical biosensor strip was demonstrated by the detection of JEV in human serum.
\end{abstract}

\section{Introduction}

Japanese Encephalitis (JE) is an infectious disease caused by flavivirus viruses that affect human's brain membrane, and it is an endemic in China, India, and Southeast Asia $[1,2]$. Currently, the treatment options for Japanese Encephalitis Virus (JEV) are limited and not often successful. Early diagnosis is therefore critical in controlling JE outbreak and preventing the development of long-term serious symptoms in patients $[3,4]$. The conventional diagnostic methods for JEV include Enzyme-Linked Immunosorbent Assays (ELISA), Plaque Reduction Neutralisation Test, Reverse Transcription Polymerase Chain Reaction (RT-PCR), and virus isolation $[1,2,5]$. However, these methods require costly and bulky laboratory facilities, tedious diagnostic procedures, specially trained personnel, and long assay time.

Most JE cases happen at rural settings in Southern and Eastern Asian countries, where diagnostic laboratory facilities are very limited [6]. A portable diagnostic system that can provide point-of-care, rapid, sensitive detection of
JEV is highly desirable in such settings [7]. Electrochemical biosensors have received much attention as a reliable diagnostic tool for infectious diseases as their sensing performances are not affected by turbidity or absorbance of the sample [8]. Besides, electrochemical biosensors offer the advantages of being highly sensitive, rapid in signal generation and readout, highly amenable for miniaturization, and inexpensive for virus detection, and it requires relatively simple operational instrumentation [9].

To date, very few studies on electrochemical biosensors for JE diagnosis have been reported. Recently, an electrochemical biosensor was fabricated for rapid detection of JE antigen by immobilized serum antibody on a silanized surface of an interdigitated sensor with a detection limit of $0.75 \mu \mathrm{gmL}^{-1}$ within 20 minutes [10]. In another study, goldcoated magnetite beads were used for the immobilization of JEV antibody and multiwall carbon nanotubes (MW-CNT) were used to improve the signal as well as the conductivity of electrochemical biosensors. However, this method suffers 
from setbacks of requiring expensive precursor materials and electrode regeneration process prior to every single diagnosis [11]. Polyaniline nanowires modified interdigitated platinum $(\mathrm{Pt})$ microelectrode electrochemical immunosensor with a detection limit of less than $10 \mathrm{ngml}^{-1}$ has also been reported. Nonetheless, the interdigitated platinum (Pt) microelectrode used was not disposable, thus suffering a setback of crosscontamination problem [5].

Many studies have attempted to incorporate various types of nanoparticles such as silver nanoparticles, gold nanoparticles, graphene, and carbon nanotubes into electrodes of biosensors in order to enhance the detection sensitivity and response time of biosensors [12-14]. Nanoparticles afford very high surface area to volume ratio, which leads to substantial increase in the loading of recognition elements on the electrodes and more efficient electrons transfer, and, in turn, enhances the sensitivity of the biosensors. However, these attempts were made on expensive precursor materials for the synthesis of nanoparticles, resulting in overall higher production costs and, therefore, low affordability for practical applications. Moreover, disposal of metal (e.g., gold, silver) nanoparticles long-term environmental effects has not been investigated so far. On the other hand, carbon nanoparticles (CNPs) have not been explored extensively for electrochemical biosensor fabrication. Carbon nanoparticles offer several advantages such as excellent electrochemical properties, inertness, low cost, low toxicity, and good chemical stability [15-18]. Herein, we report an electrochemical biosensor strip based on carbon nanoparticles modified SPCE for detection of Japanese Encephalitis Virus (JEV) with high sensitivity and selectivity. The clinical applicability of this electrochemical biosensor strip for the detection of JEV in human serum samples has been demonstrated.

\section{Materials and Methods}

2.1. Chemicals. All chemicals were of reagent grade and used without further purification. Sodium tripolyphosphate (TPP) of technical grade (85\%), bovine serum albumin (BSA), N(3-dimethylaminopropyl)- $\mathrm{N}^{\prime}$-ethylcarbodiimide hydrochloride (EDC), and N-hydroxysuccinimide 98\% (NHS) were supplied by Sigma-Aldrich (St. Louis, USA). Acetic acid was from HmbG Chemicals (Hamburg, Germany), chitosan powder with molecular weight of $100-300 \mathrm{kDa}$ was purchased from Acros Organics (New Jersey, USA), sulphuric acid of technical grade (95\%) was purchased from J. T. Baker (Thailand), phosphate buffer saline (PBS, $\mathrm{pH}$ 7) was obtained from Merck (Germany), and potassium ferricyanide $\left(\mathrm{K}_{3}\left[\mathrm{Fe}(\mathrm{CN})_{6}\right]\right)$ and potassium ferrocyanide $\left(\mathrm{K}_{4}\left[\mathrm{Fe}(\mathrm{CN})_{6}\right]\right)$ were obtained from Bendosen (Bendosen Laboratory Chemicals, Bendosen, Norway). Screen-printed carbon electrodes (SPCE) with a carbon-based working electrode, carbon-based counterelectrode, and a silver/silver chloride $(\mathrm{Ag} / \mathrm{AgCl})$ reference electrode was purchased from a local vendor, Rapid Genesis Sdn Bhd. Ultrapure water $(18.2 \mathrm{M} \Omega \cdot \mathrm{cm})$ was obtained from a water purifying system (ELGA Model: Ultra Genetic). JEV E specific monoclonal antibody (Clone ID: MV12/1/C2-2/1), JEV antigen, and JEV positive and JEV negative human serums clinical samples were provided by the Institute of Health and Community Medicine (IHCM), Universiti Malaysia Sarawak, and these biological products were stored at $-20^{\circ} \mathrm{C}$ before use.

2.2. Synthesis of Carbon Nanoparticles (CNPs). Carbon nanoparticles (CNPs) were prepared from preformed chitosan nanoparticles. The chitosan nanoparticles were prepared by crosslinking chitosan with tripolyphosphate [19].

$0.3 \mathrm{~g}$ of chitosan powder was dissolved in $2 \%(\mathrm{v} / \mathrm{v}) 100 \mathrm{~mL}$ acetic acid glacial under stirring at room temperature to obtain $0.3 \%(\mathrm{w} / \mathrm{v})$ chitosan solution. Sodium tripolyphosphate (TPP) $(1 \%(\mathrm{w} / \mathrm{v}))$ was used as an ionic crosslinker. Chitosan nanoparticles were obtained upon the addition of $1 \mathrm{~mL}$ of TPP solution into $10 \mathrm{~mL}$ of chitosan solution under sonication at room temperature for 1 hour.

$8 \mathrm{~mL}$ of concentrated sulphuric acid was then added to the chitosan nanoparticle suspension and the resulting mixture was refluxed at $180^{\circ} \mathrm{C}$ for 30 minutes by using a paraffin oil bath. The black CNPs suspension was allowed to cool down to room temperature, $40 \mathrm{~mL}$ of ultrapure water was added, and the black CNPs were collected by centrifugation (13,500 rpm).

2.3. Isolation of JEV. The Nakayama strain JEV was originally passaged from suckling mouse brain preparations and was subsequently maintained by passaging through mosquito cells, C6/36. The domain III region was cloned into a commercially available pET-SUMO vector (Invitrogen) and was expressed as fusion protein with polyhistidine in E. coli cells, BL21-DE3 with isopropyl-B-D thiogalactopyranoside (IPTG) induction. The JEV was purified using a $\mathrm{Ni}^{2+}$ chelating chromatographic column under denaturing condition and then dialyzed into phosphate buffer saline $[20,21]$.

2.4. Activation of the JEV Antibody and Immobilizing the Antibody on Surface of CNPs. A mouse monoclonal antibody (Clone ID: MV12/1/C2-2/1), specific to JEV was originally provided by Venture Technology, Penang, Malaysia [20, 21], and treated with ethyl(dimethylaminopropyl) carbodiimide (EDC) and N-hydroxysuccinimide (NHS) for activation. $200 \mu \mathrm{L}$ each of ethyl(dimethylaminopropyl) carbodiimide (EDC, 0.2 M) and N-hydroxysuccinimide (NHS, 0.05 M) were added to $200 \mu \mathrm{L}$ of JEV antibody $(10.0 \mathrm{M})$ to activate its carboxyl groups by allowing them to react for 1 hour at $4^{\circ} \mathrm{C}$. The activated JEV antibody was then added onto the surfaces of CNPs at room temperature and then incubated for 24 hours to immobilize the JEV antibody on CNPs surfaces by allowing the formation of amide bonds between amino groups on CNPs surface and activated carboxyl groups on the JEV antibody.

2.5. Instrumentation. The morphology of samples was observed using a scanning electron microscope (SEM) (JEOL JSM-6390 LA). Samples were coated with a thin layer of platinum prior to SEM analysis. FTIR spectra of samples were obtained from $\mathrm{KBr}$ sample pellets within the range of $400-4000 \mathrm{~cm}^{-1}$ on a FTIR spectrometer (Thermo Scientific, Nicolet iS10). Cyclic voltammetry 


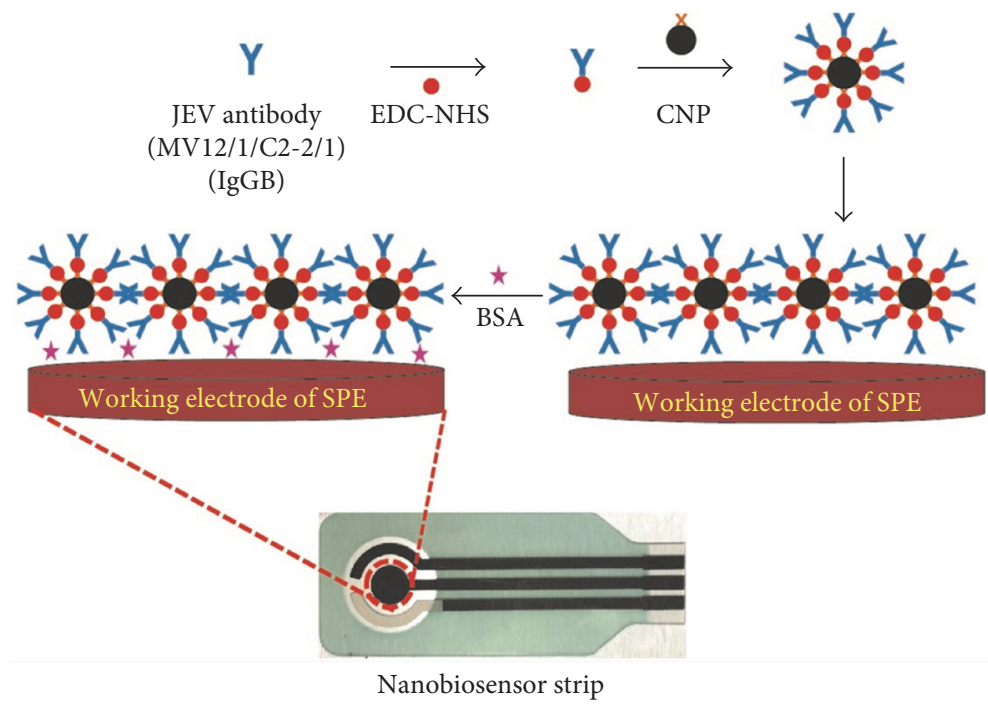

FIGURE 1: Schematic diagram of fabrication processes employed on the SPCE strip for fabricating electrochemical biosensor for the detection of JEV.

(CV) and electrochemical impedance spectroscopy (EIS) measurements were performed on a potentiostat (Princeton Applied Research, PARSTAT 2263) using an electrolyte containing $10.0 \mathrm{mM}\left[\mathrm{Fe}(\mathrm{CN})_{6}\right]^{3-/ 4-}$ and $0.1 \mathrm{M} \mathrm{KCl}$. For $\mathrm{CV}$ characterization, the potential was cycled between $-1.3 \mathrm{~V}$ and $+1.0 \mathrm{~V}$ (versus $\mathrm{Ag} / \mathrm{AgCl}$ ) at a scan rate of $50.0 \mathrm{mVs}^{-1}$. The EIS measurements were recorded within the frequency range of $0.01 \mathrm{~Hz}$ to $100.0 \mathrm{kHz}$.

2.6. Fabrication of SPCE Electrochemical Biosensor Strip. $10 \mu \mathrm{L}$ of JEV antibody immobilized CNPs was droppedcoated onto the working electrode of the SPCE strip and incubated for 48 hours at $37^{\circ} \mathrm{C}$ via physically binding mechanism, and then $2 \%(\mathrm{w} / \mathrm{v})$ bovine serum albumin (BSA) in PBS was added onto the surface of the working electrode to block unsaturated and nonspecific binding sites. The electrode was rinsed with PBS to remove excess BSA. Figure 1 illustrates a schematic diagram of the fabrication processes employed on the SPCE strip to fabricate electrochemical biosensor for the detection of JEV.

2.7. Detection of JEV Antigens. For detection of JEV antigens, $10 \mu \mathrm{L}$ of JEV of various concentrations ranging from 1 to $20.0 \mathrm{ngmL}^{-1}$ was dropped onto the CNPs modified SPCE electrochemical biosensor strip, incubated at $37^{\circ} \mathrm{C}$ for $20 \mathrm{~min}$, rinsed, and air-dried. The interaction of JEV with antibody immobilized on the surface of CNPs modified SPCE electrochemical biosensor strip working electrode was studied using EIS. The relative resistance was calculated based on the following equation [22]:

$$
\text { relative resistance }=\frac{R_{\mathrm{et}}(i)-R_{\mathrm{et}}(o)}{R_{\mathrm{et}}(o)},
$$

where $R_{\mathrm{et}}(i)$ is the value of electron transfer resistance after the binding of JEV to JEV antibody and $R_{\text {et }}(o)$ is the value of electron transfer resistance after the immobilization of
JEV antibody onto CNPs modified SPCE electrochemical biosensor strip working electrode surface and block with BSA.

\section{Results and Discussion}

Scanning electron micrographs (SEM) of chitosan nanoparticles and carbon nanoparticles (CNPs) used for immobilizing antibody on the SPCE strip were shown in Figures 2(a) and 2(b), respectively. The mean particle size of the CNPs was around $100 \mathrm{~nm}$. CNPs provide a large specific surface area for JEV antibody immobilization while enhancing the efficiency of electron transfer. Moreover, the amino group on the surface of CNPs can be used for attachment of antibody.

A distinctive peak at $1619 \mathrm{~cm}^{-1}$ due to bending vibration of $\mathrm{N}-\mathrm{H}$ in the amino group [15] was observed in the Fourier Transform Infrared (FTIR) spectrum of CNPs as shown in Figure 3(a). This indicated that amino groups on chitosan nanoparticles remained after the carbonization process. The amino groups on surfaces of CNPs were used to form amide bonds with carboxyl groups of JEV antibody through coupling reaction leading to immobilization of the antibody on the CNPs [23]. The activated carboxyl groups of JEV antibody underwent coupling reaction after being dropped on the surface of CNPs at room temperature. The presence of a characteristic carbonyl $(\mathrm{C}=\mathrm{O})$ stretching of amide bonds absorption peak at around $1631 \mathrm{~cm}^{-1}$ [24] and the narrowing band at $3422 \mathrm{~cm}^{-1}$ due to the disappearance of carboxylic acid groups in the FTIR spectrum of the JEV antibody immobilized confirmed the formation of amide bonds between amino groups of CNPs and carboxylic groups of JEV antibody, Figure 3(b).

The JEV antibody immobilized CNPs modified SPCE electrochemical biosensor strip was characterized by cyclic voltammetry in the presence of $10 \mathrm{mM}$ $\mathrm{K}_{3} \mathrm{Fe}(\mathrm{CN})_{6} / \mathrm{K}_{4} \mathrm{Fe}(\mathrm{CN})_{6}(1: 1)$ as redox probes and $0.1 \mathrm{M}$ 


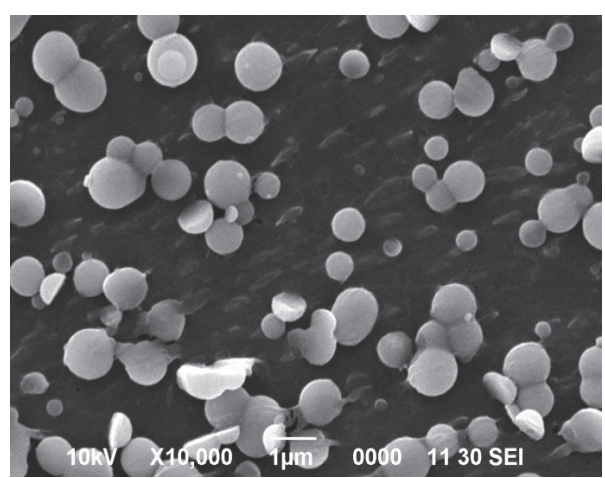

(a)

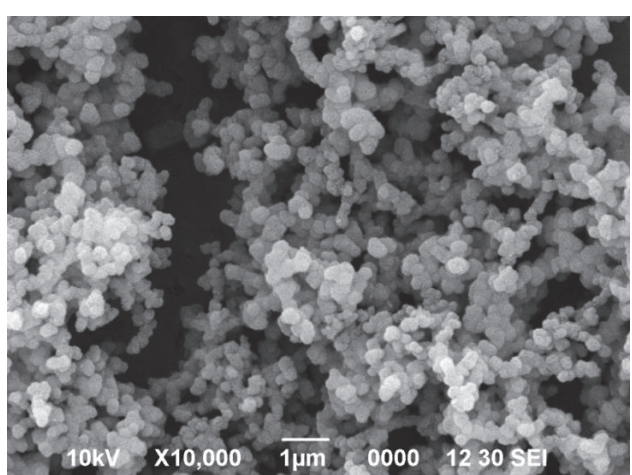

(b)

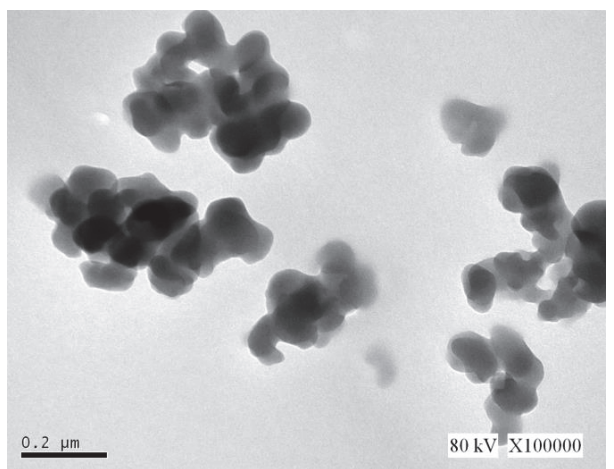

(c)

FIGURE 2: Scanning electron micrographs of (a) chitosan nanoparticles and (b) carbon nanoparticles (CNPs); (c) transmission electron micrograph of carbon nanoparticles used for the immobilization of JEV antibody.

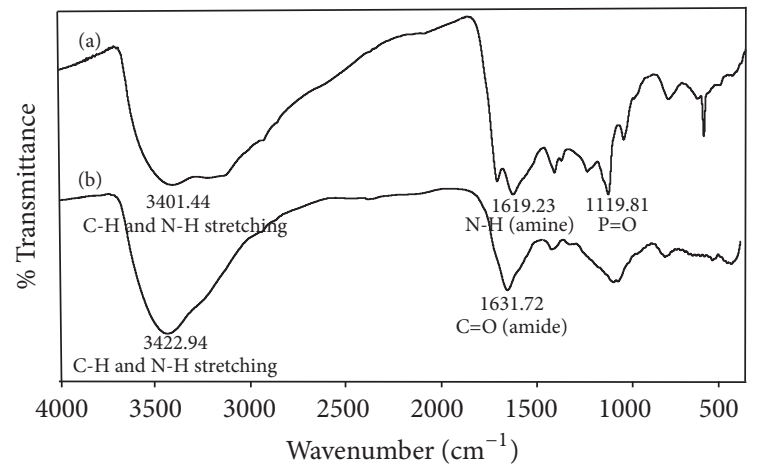

FIGURE 3: FTIR spectra of (a) carbon nanoparticles (CNPs) and (b) JEV antibody immobilized onto carbon nanoparticles.

$\mathrm{KCl}$ at $\mathrm{pH} \sim$ 7.4. Cyclic voltammogram in Figure 4(a) showed that CNPs modified SPCE electrochemical biosensor strip (curve (i)) exhibited about 9.2\% higher cathodic current density than unmodified SPCE strip (curve (ii)). The presence of CNPs led to increase in the conductivity and effective electroactive surface area of SPCE. Addition of JEV antibody immobilized CNPs onto SPCE strip resulted in an observable decrease in cathodic current (curve (iii)). The magnitude of cathodic current was further decreased upon addition of BSA (curve (iv)) due to the blocking of nonspecific sites present on the working electrode that hindered electrons transfer. In the presence of JEV, the cathodic current was observed to drop further (curve (v)). The presence of JEV was detected based on the highly specific recognition between JEV and the antibody immobilized on the CNPs. The formation of antigen-antibody complexes insulated the electrode by hindering the redox reaction of $\mathrm{K}_{3} \mathrm{Fe}(\mathrm{CN})_{6} / \mathrm{K}_{4} \mathrm{Fe}(\mathrm{CN})_{6}$ and diffusion towards the electrode surface which led to the observed decrease in cathodic current (curve (v)).

Electrochemical impedance spectroscopy (EIS) measurements were carried out in the presence of $10.0 \mathrm{mM}$ of $\mathrm{K}_{3} \mathrm{Fe}(\mathrm{CN})_{6} / \mathrm{K}_{4} \mathrm{Fe}(\mathrm{CN})_{6}(\mathrm{pH} \sim 7.4)$ containing $0.1 \mathrm{M} \mathrm{KCl}$ in order to further investigate the effects of electron transfer at the electrode surface. All EIS data were simulated by the Randles circuit as shown in the inset of Figure 4(b). As observed in Figure 4(b), the Nyquist plot of unmodified SPCE electrochemical biosensor strip displayed a larger semicircle of larger diameter (curve (i)) than that of CNPs modified SPCE electrochemical biosensor strip (curve (ii)). Unmodified SPCE had exhibited a higher electron transfer resistance $\left(R_{\mathrm{et}}=764 \mathrm{ohm}\right)$ as compared with CNPs modified $\operatorname{SPCE}\left(R_{\mathrm{et}}=660 \mathrm{ohm}\right)$ due to faster electron transfer rates at the electrode surface. The immobilization of JEV antibody onto the surface of CNPs had resulted in an increase in $R_{\mathrm{et}}$ (curve (iii)) due to the presence of the hydrophobic layer of an antibody which hindered interfacial electron transfer. As expected, $R_{\text {et }}$ was observed to increase after the addition 


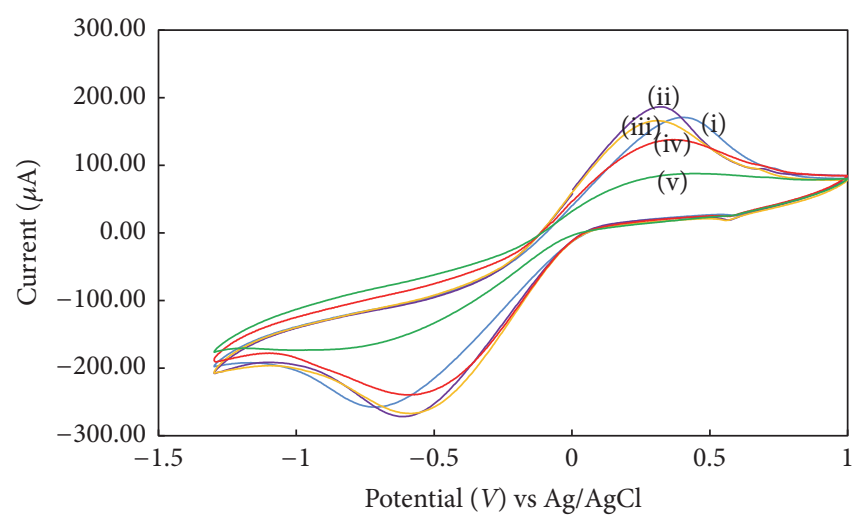

(a)

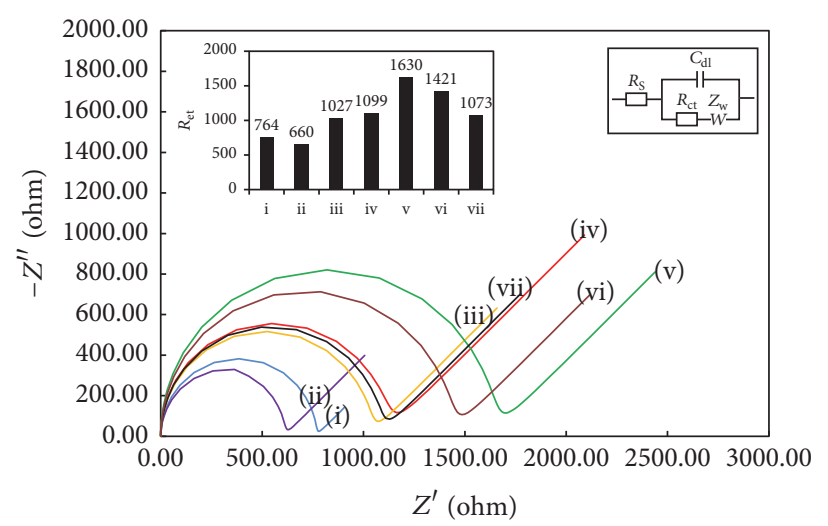

(b)

FIGURE 4: (a) Cyclic voltammograms (CV) and (b) Nyquist plots of (i) bare SPCE strip; (ii) CNPs modified SPCE strip; (iii) JEV antibody immobilized CNPs on SPE strip; (iv) BSA blocked/JEV antibody immobilized CNPs on SPCE strip; (v) BSA blocked/JEV antibody immobilized CNPs on SPCE strip in the presence of JEV; (vi) JEV positive human serum; (vii) Dengue antigen. Both CV and EIS were conducted in PBS solution ( $\mathrm{pH} 7.4$ ) containing $0.1 \mathrm{M} \mathrm{KCl}$ and $15 \mathrm{mM}$ of $\mathrm{K}_{3}\left[\mathrm{Fe}(\mathrm{CN})_{6}\right] / \mathrm{K}_{4}\left[\mathrm{Fe}(\mathrm{CN})_{6}\right]$ at a scan rate of $50 \mathrm{mVs}^{-1}$ and frequency range of $0.01 \mathrm{~Hz}$ to $100.0 \mathrm{kHz}$, respectively. [Inset of $4 \mathrm{~B}$ shows electron transfer resistance, $R_{\mathrm{et}}$, for various Nyquist plots (i-vii) and the Randles equivalent circuit.]

of BSA to block nonspecific sites (curve (iv)). In the presence of JEV, the $R_{\text {et }}$ was observed to further increase (curve (v)) which was attributed to the formation of the antigenantibody nonconductive layer which blocked electron transfer at the electrode surface.

In order to demonstrate the clinical applicability and selectivity of the CNPs modified SPCE electrochemical biosensor strip, both JEV positive human serum clinical samples and Dengue antigen were used as analytes and evaluated by using EIS. As observed in Figure 4(b), in the presence of sample containing JEV positive human serum (curve (vi)), the $R_{\text {et }}(1421 \mathrm{ohm})$ was observed to be substantially higher as compared to the $R_{\text {et }}(1099 \mathrm{ohm})$ of BSA blocked/JEV antibody immobilized CNPs on SPCE strips (curve (iv)). These results indicated that the CNPs modified SPCE electrochemical biosensor strip was capable of detecting JEV in human serums. The selectivity of this biosensor strip was proven as in the presence of Dengue antigen; the $R_{\mathrm{et}}(1073 \mathrm{ohm})$ of (curve (vii)) was observed to have no significant changes as compared with $R_{\text {et }}(1099 \mathrm{ohm})$ of BSA blocked/JEV antibody immobilized CNPs on SPCE strips (curve (iv)). This is because Dengue antigen was unable to form an antigen-antibody nonconductive layer with the antibody of JEV.

The EIS spectra of CNPs modified SPCE electrochemical biosensor strip incubated with various concentrations of JEV were displayed in Figure 5. The diameters of semicircles were observed to increase as the concentration of JEV was increased. The inset of Figure 5 shows the calibration plots of relative resistance calculated by (1) versus concentrations of JEV ranging from 1 to $20 \mathrm{ngmL}^{-1}$; the relative resistance increased linearly $\left(R^{2}=0.913\right)$ with increase in JEV concentration. The observed increase in $R_{\mathrm{et}}$ could be attributed to the formation of more antigen-antibody complexes at higher JEV concentration which inhibited the interfacial electron

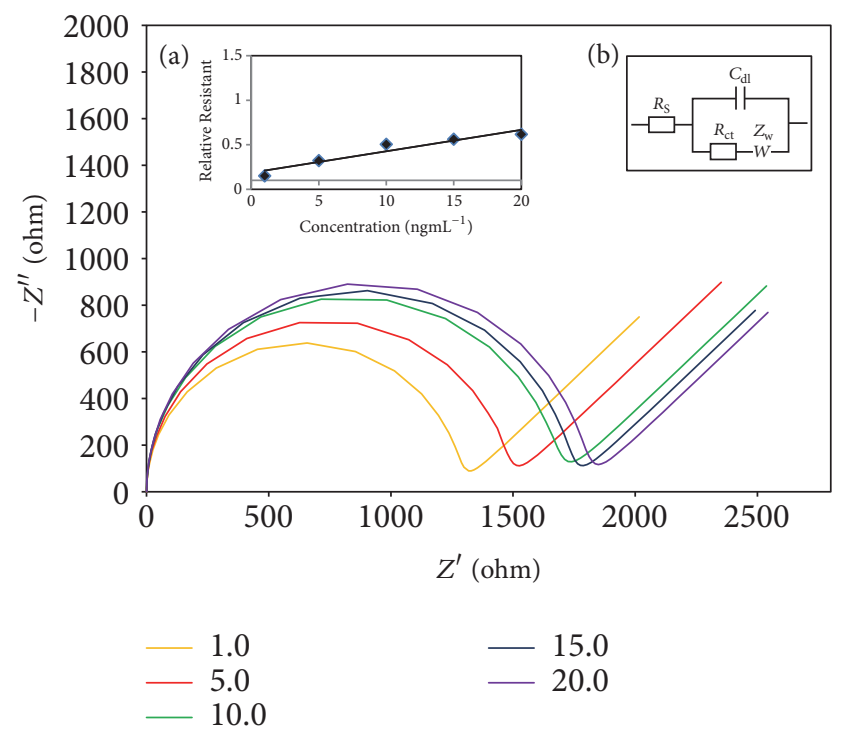

FIGURE 5: Nyquist plots of JEV antibody immobilized CNPs on SPCE strip with different JEV concentrations ( $\mathrm{ng} / \mathrm{mL}$ ). Inset (a) shows calibration plots of relative resistance versus concentrations of JEV ranging from 1 to $20 \mathrm{ngmL}^{-1}$ and (b) the Randles equivalent circuit. EIS was conducted in $10 \mathrm{mM}$ of $\mathrm{K}_{3}\left[\mathrm{Fe}(\mathrm{CN})_{6}\right] / \mathrm{K}_{4}\left[\mathrm{Fe}(\mathrm{CN})_{6}\right]$ ( $\mathrm{pH} \sim 7.4$ ) containing $0.1 \mathrm{M} \mathrm{KCl}$ at a scan rate of $50 \mathrm{mVs}^{-1}$ within the frequency range of $0.01 \mathrm{~Hz}$ to $100.0 \mathrm{kHz}$. (Error bars of insets were calculated from the mean value, $\mathrm{s} / \mathrm{n}=3$.)

transfer process. A linear correlation equation between relative resistance and JEV concentration $\left(\mathrm{ngmL}^{-1}\right)$ was obtained (inset of Figure 5) as shown in

$$
\text { relative resistance }=0.024 \mathrm{Conc} \mathrm{JEV}+0.186 \text {. }
$$

Conc. JEV is the concentration of JEV in $\mathrm{ngmL}^{-1}$. The values of regression coefficient $\left(R^{2}\right)$ and standard deviation $(\sigma)$ were 


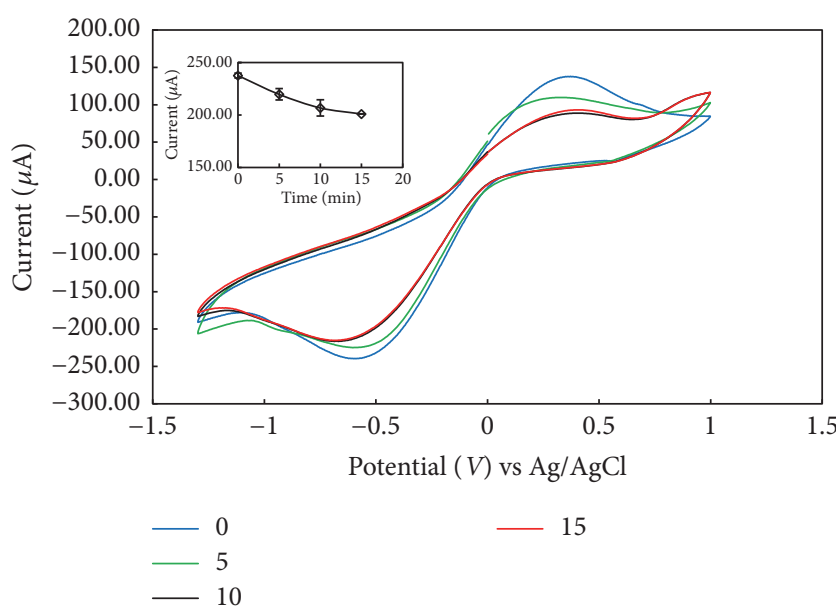

FIGURE 6: Cyclic voltammograms of JEV antibody immobilized CNPs on SPCE biosensor strip as a function of response time: (a) $0 \mathrm{~min}$; (b) $5 \mathrm{~min}$; (c) $10 \mathrm{~min}$; (d) $15 \mathrm{~min}$. The inset shows the plot of current $(\mu \mathrm{A})$ versus response time $(\mathrm{min})$.

0.91 and 0.19 , respectively. The calibration plot of relative resistance versus concentrations of JEV ranging from 1 to $20 \mathrm{ngmL}^{-1}$ was used to determine the limit of detection (LOD) for the biosensor strip. The SPCE electrochemical biosensor strip showed a very low detection limit of $0.36 \mathrm{ngmL}^{-1}$ for JEV. From (2), the detection sensitivity was $0.024 \mathrm{ngmL}^{-1}$. This detection limit is substantially lower than the previously reported detection limit of $10 \mathrm{ngmL}^{-1}$ for an interdigitated sensor [1] and a polyaniline nanowires based electrochemical immunosensor at below $10 \mathrm{ngmL}^{-1}$ [5]. The CNPs modified SPCE biosensor reported in this work exhibited more than an order of magnitude higher in sensitivity as compared to the unmodified SPCE biosensor for JEV detection.

Cyclic voltammograms of CNPs modified SPCE electrochemical biosensor strip as a function of response time were shown in Figure 6. The CNPs modified SPCE electrochemical biosensor strip was observed to achieve stable cathodic current in about 10 minutes. This result demonstrated that the proposed biosensor strip exhibited rapid responses, and results of the analysis were obtainable within 10 minutes. This observed response time is considerably faster than that reported for interdigitated biosensor at 20 minutes [10] and other JEV diagnosis methods which require diagnosis times ranging from hours to days [14]. The substantially enhanced sensitivity and faster response time could be attributed to the use of CNPs which enabled a higher loading of JEV antibody as well as the increased overall electrical conductivity of the SPCE biosensor strip.

\section{Conclusion}

In this work, a disposable CNPs modified SPCE electrochemical biosensor strip was successfully fabricated for rapid and sensitive detection of JEV. This biosensor strip can achieve stable response signal within 10 minutes and shows a limit of detection of $0.36 \mathrm{ngmL}^{-1}$ and detection sensitivity was
$0.024 \mathrm{ngmL}^{-1}$ for JEV. The disposable nature of this biosensor strip will prevent cross-contamination from previous samples in clinical diagnosis. The potential clinical application of this biosensor strip for detecting JEV in human serum samples has been demonstrated.

\section{Conflicts of Interest}

The authors declare that there are no conflicts of interest regarding the publication of this paper. The authors of this paper have no direct financial relation with the commercial entities mentioned in this paper.

\section{Acknowledgments}

The authors gratefully acknowledge the financial support by the Ministry of Education Malaysia (KPM) rendered through the award of the Exploratory Research Grant Scheme ERGS/STG05(01)/1005/2013(02) as well as a scholarship from the Ph.D. of one of the authors.

\section{References}

[1] Q. H. Tran, T. H. H. Nguyen, A. T. Mai, T. T. Nguyen, Q. K. $\mathrm{Vu}$, and T. N. Phan, "Development of electrochemical immunosensors based on different serum antibody immobilization methods for detection of Japanese encephalitis virus," Advances in Natural Sciences: Nanoscience and Nanotechnology, vol. 3, Article ID 015012, 2012.

[2] M. Bonaparte, B. Dweik, E. Feroldi et al., "Immune response to live-attenuated Japanese encephalitis vaccine (JE-CV) neutralizes Japanese encephalitis virus isolates from South-East Asia and India," BMC Infectious Diseases, vol. 14, no. 1, article 156, 2014.

[3] J. Hombach, T. Solomon, I. Kurane, J. Jacobson, and D. Wood, "Report on a WHO consultation on immunological endpoints for evaluation of new Japanese encephalitis vaccines, WHO, Geneva, 2-3 September, 2004," Vaccine, vol. 23, no. 45, pp. 52055211, 2005.

[4] Q. H. Tran, A. T. Mai, T. T. Nguyen, and T. H. H. Nguyen, "Towards the use of protein A-tagged gold nanoparticles for signal amplification of electrochemical immunosensors in virus detection," Advances in Natural Sciences: Nanoscience and Nanotechnology, vol. 3, Article ID 025013, 2012.

[5] C. V. Tuan, T. Q. Huy, N. V. Hieu, M. A. Tuan, and T. Trung, "Polyaniline nanowires-based electrochemical immunosensor for label free detection of japanese encephalitis virus," Analytical Letters, vol. 46, no. 8, pp. 1229-1240, 2013.

[6] R. J. Coker, B. M. Hunter, J. W. Rudge, M. Liverani, and P. Hanvoravongchai, "Emerging infectious diseases in southeast Asia: regional challenges to control," The Lancet, vol. 377, no. 9765, pp. 599-609, 2011.

[7] K. E. Jones, N. G. Patel, M. A. Levy et al., "Global trends in emerging infectious diseases," Nature, vol. 451, Article ID 06536, pp. 990-993, 2008.

[8] E. Bakker and M. Telting-Diaz, "Electrochemical sensors," Analytical Chemistry, vol. 74, no. 12, pp. 2781-2800, 2002.

[9] B. Pejcic, R. D. Marco, and G. Parkinson, "The role of biosensors in the detection of emerging infectious diseases," Analyst, vol. 131, no. 10, pp. 1079-1090, 2006. 
[10] T. Q. Huy, N. T. H. Hanh, N. T. Thuy, P. V. Chung, P. T. Nga, and M. A. Tuan, "A novel biosensor based on serum antibody immobilization for rapid detection of viral antigens," Talanta, vol. 86, no. 1, pp. 271-277, 2011.

[11] F. Li, L. Mei, Y. Li et al., "Facile fabrication of magnetic gold electrode for magnetic beads-based electrochemical immunoassay: Application to the diagnosis of Japanese encephalitis virus," Biosensors and Bioelectronics, vol. 26, no. 10, pp. 4253-4256, 2011.

[12] K. J. Huang, J. Li, Y. M. Liu, X. Cao, S. Yu, and M. Yu, "Disposable immunoassay for hepatitis B surface antigen based on a graphene paste electrode functionalized with gold nanoparticles and a Nafion-cysteine conjugate," in Microchim. Acta, vol. 177, pp. 419-426, 2012.

[13] S. C. Tan, S. F. Chin, and S. C. Pang, "Disposable carbon dots modified screen printed carbon electrode electrochemical sensor strip for selective detection of ferric ions," Journal of Sensors, vol. 2017, Article ID 7576345, 7 pages, 2017.

[14] Z. Taleat, A. Khoshroo, and M. Mazloum-Ardakani, "Screenprinted electrodes for biosensing: a review (2008-2013)," Microchimica Acta, vol. 181, no. 9-10, pp. 865-891, 2014.

[15] S. F. Chin, S. C. Tan, S. C. Pang, and S. M. Ng, "Nitrogen-doped carbon nanodots as fluorescent probes for selective detection and quantification of Ferric (III) ions," Optical Materials, vol. 73, pp. 77-82, 2017.

[16] Y. H. Ng, S. F. Chin, S. C. Pang, and S. M. Ng, "The luminescence profile of carbon dots synthesized from a-cellulose under different acid hydrolysis conditions," Optical Materials, vol. 70, pp. 50-56, 2017.

[17] J. F. Y. Fong, S. F. Chin, and S. M. Ng, "A unique, turn-on , fluorescence signaling strategy for highly specific detection of ascorbic acid using carbon dots as sensing probe," in Biosens. Bioelectron, vol. 85, pp. 844-852, 2016.

[18] S. F. Chin, L. S. Lim, S. C. Pang, M. S. H. Sum, and D. Perera, "Carbon nanoparticle modified screen printed carbon electrode as a disposable electrochemical immunosensor strip for the detection of Japanese encephalitis virus," Microchimica Acta, vol. 184, no. 2, pp. 491-497, 2017.

[19] W. Fan, W. Yan, Z. Xu, and H. Ni, "Formation mechanism of monodisperse, low molecular weight chitosan nanoparticles by ionic gelation technique," in in Colloids Surf, vol. 90, pp. 21-27, B, 2012.

[20] M. J. Cardosa, S. M. Wang, M. S. H. Sum, and P. H. Tio, "Antibodies against prM protein distinguish between previous infection with dengue and Japanese encephalitis viruses," $B M C$ Microbiology, vol. 2, no. 1, 2002.

[21] M. S. H. Sum, A. Andrew, and M. A. Maling, "Expression of recombinant E1 glycoprotein of chikungunya virus in baculovirus expression system," Journal of Biochemistry, Microbiology and Biotechnology, vol. 3, pp. 26-29, 2015.

[22] B. Rezaei, M. Saghebdoust, A. M. Sorkhe, and N. Majidi, "Generation of a doxorubicin immunosensor based on a specific monoclonal antibody-nanogold-modified electrode," Electrochimica Acta, vol. 56, no. 16, pp. 5702-5706, 2011.

[23] S. Roy, N. Soin, R. Bajpai, D. S. Misra, J. A. McLaughlin, and S. S. Roy, "Graphene oxide for electrochemical sensing applications," Journal of Materials Chemistry, vol. 21, no. 38, pp. 14725-14731, 2011.

[24] A. Sharma, Z. Matharu, G. Sumana, P. R. Solanki, C. G. Kim, and B. D. Malhotra, "Antibody immobilized cysteamine functionalized-gold nanoparticles for aflatoxin detection," Thin Solid Films, vol. 519, no. 3, pp. 1213-1218, 2010. 

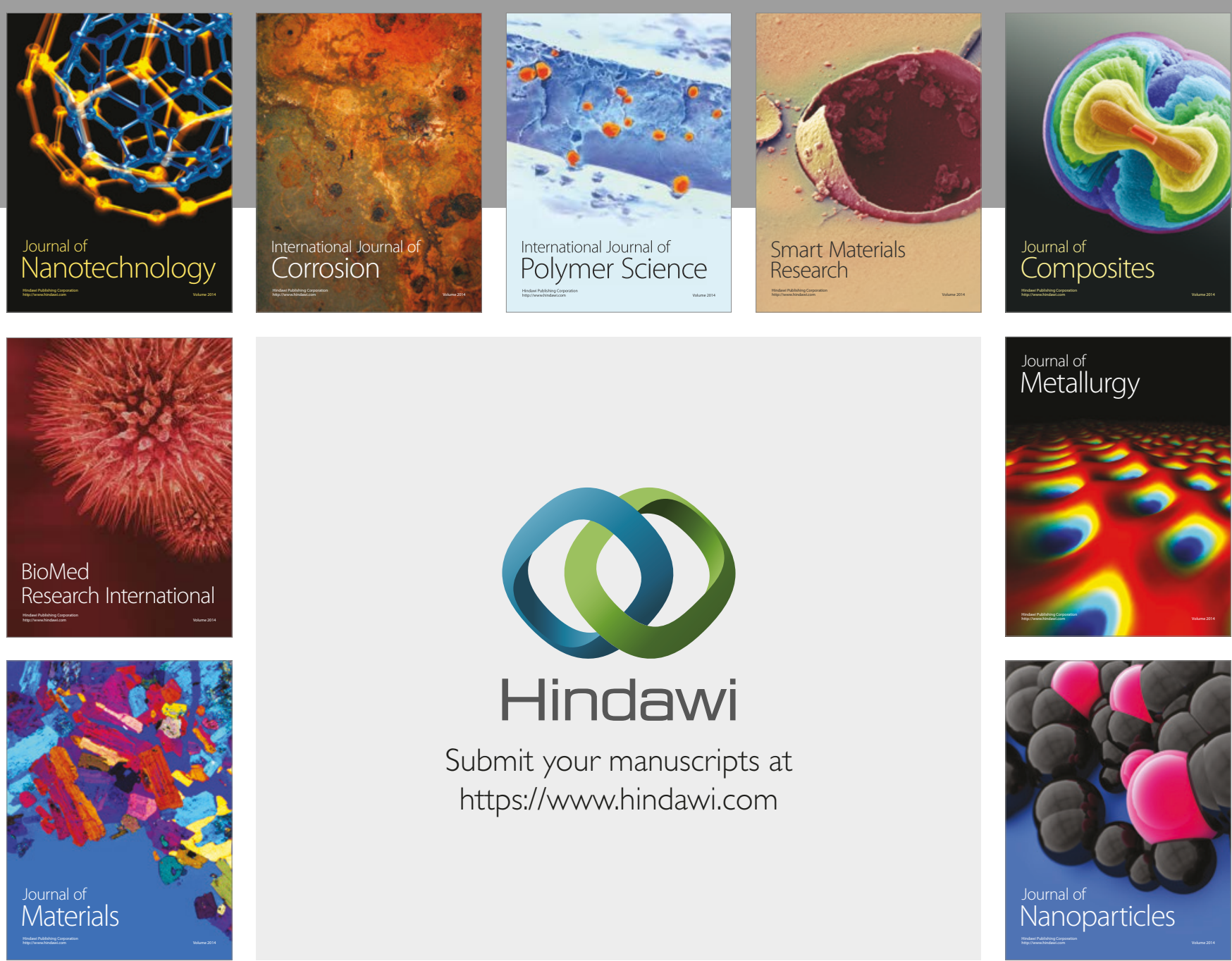

\section{Hindawi}

Submit your manuscripts at

https://www.hindawi.com
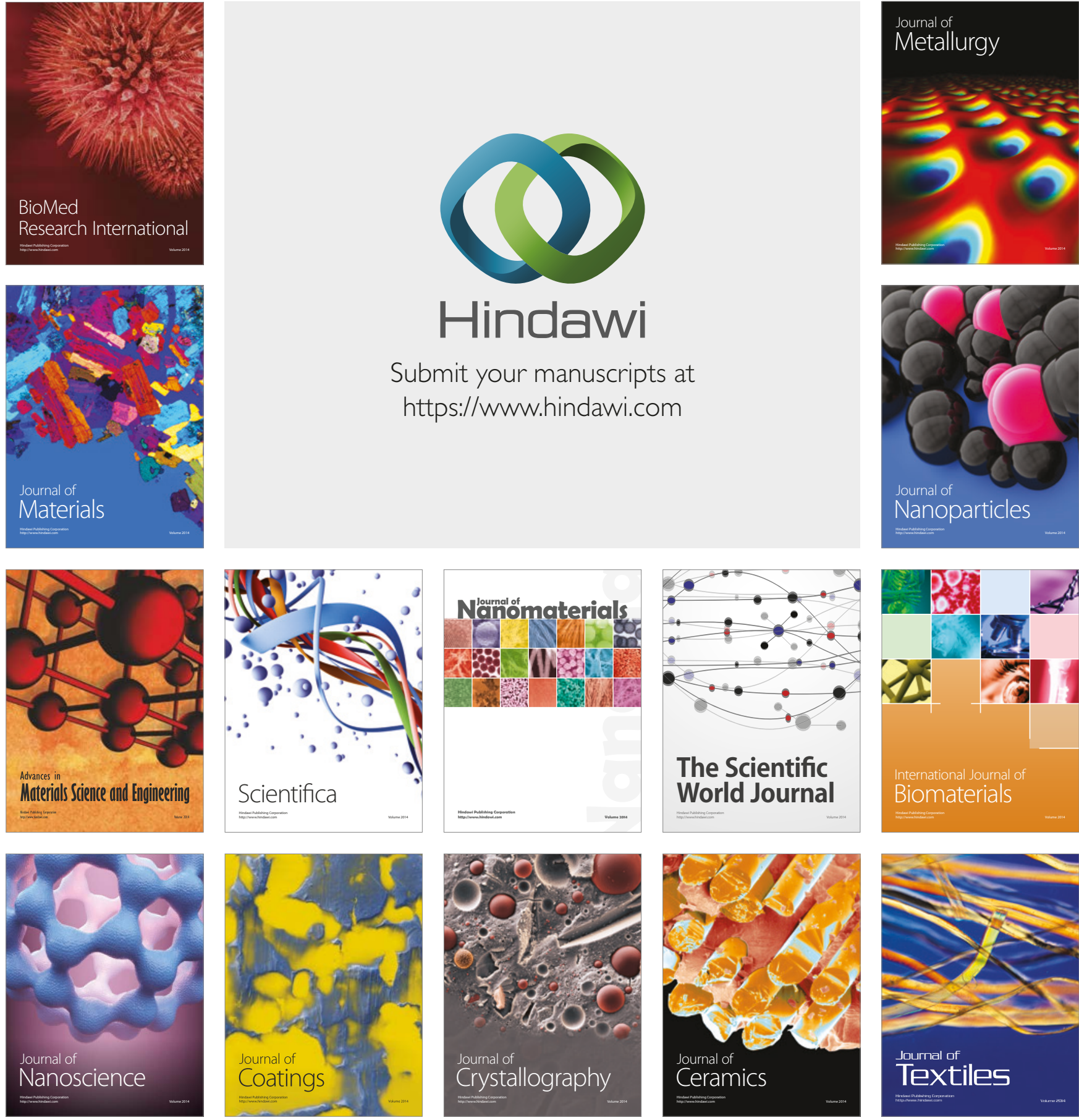

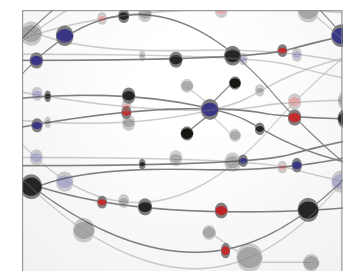

The Scientific World Journal
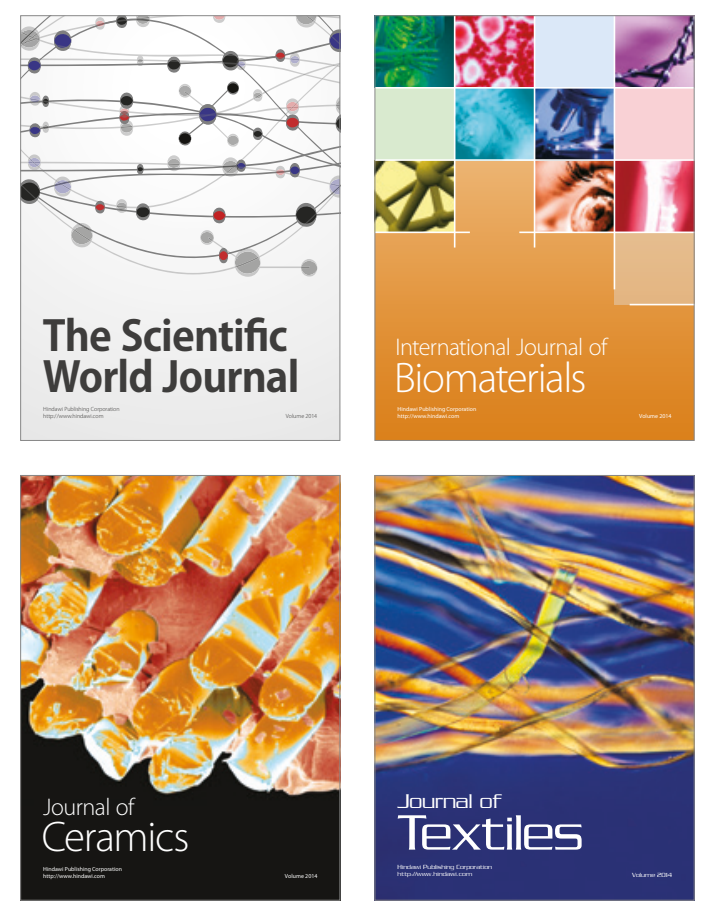